\title{
BMJ Open Adiposity, physical activity and risk of diabetes mellitus: prospective data from the population-based HUNT study, Norway
}

\author{
Kirsti Vik Hjerkind, ${ }^{1,2}$ Jo S Stenehjem, ${ }^{1,2}$ Tom I L Nilsen ${ }^{1}$
}

To cite: Hjerkind KV, Stenehjem JS, Nilsen TIL. Adiposity, physical activity and risk of diabetes mellitus: prospective data from the population-based HUNT study, Norway. BMJ Open 2017;7:e013142.

doi:10.1136/bmjopen-2016013142

- Prepublication history for this paper is available online. To view these files please visit the journal online (http://dx.doi.org/10.1136/ bmjopen-2016-013142).

Received 22 June 2016 Revised 28 September 2016 Accepted 27 October 2016

CrossMark

\footnotetext{
${ }^{1}$ Cancer Registry of Norway, Institute of Population-based Cancer Research, Oslo, Norway

${ }^{2}$ Department of Public Health, Norwegian University of Science and Technology, Trondheim, Norway
}

Correspondence to Dr Kirsti Vik Hjerkind; Kirsti. Vik.Hjerkind@kreftregisteret.no

\section{ABSTRACT}

Background: Physical activity may counteract the adverse effects of adiposity on cardiovascular mortality; however, the evidence of a similar effect on diabetes is sparse. This study examines whether physical activity may compensate for the adverse effect of adiposity on diabetes risk.

Methods: The study population consisted of 38231 individuals aged 20 years or more who participated in two consecutive waves of the prospective longitudinal Nord-Trøndelag Health Study in Norway: in 1984-1986 and in 1995-1997. A Poisson regression model with SEs derived from robust variance was used to estimate adjusted risk ratios of diabetes between categories of body mass index and physical activity.

Results: Risk of diabetes increased both with increasing body mass $\left(P_{\text {trend }}<0.001\right)$ and with decreasing physical activity level $\left(P_{\text {trend }}<0.001\right.$ in men and 0.01 in women). Combined analyses showed that men who were both obese and had low activity levels had a risk ratio of 17 (95\% Cl 9.52 to 30$)$ compared to men who were normal weight and highly active, whereas obese men who reported high activity had a risk ratio of $13(95 \% \mathrm{Cl} 6.92$ to 26$)$. Corresponding analysis in obese women produced risk ratios of 15 (95\% Cl 9.18 to 25$)$ and $13(95 \% \mathrm{Cl} 7.42$ to 21$)$ among women reporting low and high activity levels, respectively.

Conclusions: This study shows that overweight and obesity are associated with a substantially increased risk of diabetes, particularly among those who also reported being physically inactive. High levels of physical activity were associated with a lower risk of diabetes within all categories of body mass index, but there was no clear evidence that being physically active could entirely compensate for the adverse effect of adiposity on diabetes risk.

\section{INTRODUCTION}

Adiposity is the single most important predictor of diabetes, ${ }^{1-5}$ but physical inactivity has also been related to an increased risk. ${ }^{6-10}$ The protective effect of physical activity on the development of diabetes is primarily
Strengths and limitations of this study

- This is a large population-based longitudinal study of the joint association of body mass index and physical activity with diabetes risk.

- The questionnaire-based diabetes diagnoses have been validated by comparison with medical records, and were verified in $96 \%$ of the cases.

- We were unable to differentiate between type 1 and type 2 diabetes, but the ratio of type 2 vs type 1 is not likely to be differential between exposure groups.

- We lacked information on diet and family history of diabetes, and cannot exclude possible confounding from these factors.

linked to improved insulin sensitivity and glucose metabolism, ${ }^{11}$ and a reduction in body weight is not necessary for a beneficial impact on glycaemic control. ${ }^{12}$

The combined effects of body mass index (BMI) and physical activity has been investigated in relation to cardiovascular mortality, under the hypothesis that high levels of physical activity may cancel out the adverse effects of adiposity. ${ }^{13}{ }^{14}$ However, whether physical activity may compensate for the increased risk of adiposity-induced diabetes has been sparsely studied. Two previous studies have examined the joint association of adiposity and physical activity on the risk of diabetes in women only, and both reported that the influence of adiposity was larger than that of physical inactivity. 515

The objective of this study was to prospectively examine both the independent and combined effects of physical activity and adiposity on the risk of diabetes in a large prospective longitudinal study of Norwegian men and women. More specifically, we examined whether physical activity can compensate for the adverse effects of adiposity on the risk of diabetes. 


\section{METHODS}

\section{Study population}

The present study uses information from the Nord-Trøndelag Health Study (HUNT) in Norway, where all residents aged 20 years or more were invited to participate. In the first study (HUNT1, 1984-198686), 85100 people were eligible to participate and 74977 (88\%) accepted the invitation. In the second study (HUNT2, 1995-1997) 66140 (71\%) participated of 92936 eligible individuals.

Included in the study sample are the 45925 people who participated at HUNT1 and HUNT2. Of those, we excluded 606 individuals who had diabetes at baseline, 36 due to missing information on diabetes status, height or weight, and 5 since they were below 20 years of age at baseline. A total of 6772 individuals failed to answer the questionnaire that contained information on physical activity, and were thus excluded from the analysis of physical activity. Finally, 93 individuals were excluded because of missing information on the outcome variable diabetes at HUNT2. Thus, 38413 individuals (18 019 men and 20394 women) were included in analyses involving physical activity measures and 45185 individuals (21 210 men and 23975 women) were included in analyses of BMI alone.

\section{Study variables}

All participants completed comprehensive questionnaires and underwent a clinical examination. The collected data included information on lifestyle and health-related factors such as self-reported measures of physical activity, smoking, alcohol consumption and level of education, as well as clinical measurements of blood pressure, blood glucose levels, heart rate, height and weight. A detailed description of procedures and methods is found at http://www.ntnu.edu/hunt.

\section{Diabetes}

Information on the outcome variable at HUNT2, whether an individual had developed diabetes after 11 years of follow-up, was obtained from the question "Do you have or have you had diabetes?". This questionnaire-based diabetes diagnoses have been validated by comparison with medical records, and were verified in $96 \%$ of the cases. ${ }^{16}$

Exclusion of prevalent cases of diabetes at baseline (HUNT1) was based on a positive response to the same question as at HUNT2, and by blood glucose measurements for individuals aged $>40$ years. Individuals with non-fasting glucose values of $\geq 8.0 \mathrm{mmol} / \mathrm{L}$ were invited to take a fasting blood glucose test. If fasting blood glucose was $\geq 7.0 \mathrm{mmol} / \mathrm{L}$, the individual was defined as having diabetes. If fasting glucose was $<7.0 \mathrm{mmol} / \mathrm{L}$, the individual was given a glucose load (OGTT, oral glucose tolerance test) and new measurements were performed. Individuals were defined having diabetes if blood glucose after 2 hours was $\geq 11.0 \mathrm{mmol} / \mathrm{L}^{16}$

\section{Body mass index}

Standardised measurements of height (to the nearest centimetre) and weight (to the nearest half kilogram) were obtained at the clinical examination. Based on these measures, BMI was calculated as weight divided by the square value of height $\left(\mathrm{kg} / \mathrm{m}^{2}\right)$. BMI was further divided into underweight and normal weight (14.5$\left.24.9 \mathrm{~kg} / \mathrm{m}^{2}\right)$, overweight $\left(25.0-29.9 \mathrm{~kg} / \mathrm{m}^{2}\right)$ and obese (30 $\mathrm{kg} / \mathrm{m}^{2}$ or above).

\section{Physical activity}

Participants reported on recreational physical activity during an average week. Frequency was recorded as 0, $<1,1,2-3$ or $\geq 4$ times a week. In participants reporting exercise at least once a week, duration was recorded as $<15,15-30,31-60$ or $\geq 60 \mathrm{~min}$ and intensity as light, moderate or vigorous. Moderate and high intensity were collapsed in analyses to maintain sufficient statistical power. A summary score of frequency, duration and intensity was constructed according to the following equation: $1 / 5 \times$ frequency $+1 / 4 \times$ duration $+1 / 3 \times$ intensity, giving equal weight to each component. Score values ranged from 1.18 to 3.00 , and were categorised into medium or high physical activity levels based on sexspecific medians (using cut-points 2.02 for men and 1.83 for women).No activity and activity $<1$ a week were categorised as low physical activity level. The summary score has been used in previous studies using HUNT data. ${ }^{17} 18$

\section{Statistical analyses}

Risk ratios (RRs) of diabetes according to physical activity and BMI were estimated with 95\% CIs using a Poisson regression model with SEs derived from robust variance. ${ }^{19}$ Analyses were gender-specific, and results were adjusted for age (continuous), education $(<10,10-12$, $>12$ years or unknown), use of alcohol (frequency in the past 2 weeks: $0,1-4, \geq 5$, abstainer or unknown), smoking status (never, former, current or unknown), ever used blood pressure medication (yes, no or unknown) and ever suffered a cardiovascular disease (yes, no or unknown). Physical activity and BMI were mutually adjusted in the respective analyses. Trend tests across categories of physical activity and BMI were calculated by treating the categories as ordinal variables in the regression model. Interaction between physical activity and BMI was tested for by including an interaction term of these variables in the model. In supplementary analyses, we assessed whether age ( $<60$ years vs $\geq 60$ years) could modify the association between physical activity and risk of diabetes by including a product term of age and the physical activity summary score in the regression model.

All statistical tests were two-sided, and all statistical analyses were conducted using Stata, V.14.2 (StataCorp, College Station, Texas, USA).

\section{Ethics}

All participants signed a written informed consent at participation in the HUNT Study. 
Table 1 Baseline characteristics of the study population according to a physical activity summary score*

\begin{tabular}{|c|c|c|c|}
\hline \multirow[b]{2}{*}{ Characteristic } & \multicolumn{3}{|c|}{ Physical activity summary score* } \\
\hline & Low & Medium & High \\
\hline \multicolumn{4}{|l|}{ Men } \\
\hline Number of persons, $\mathrm{N}(\%)$ & $7466(35.2)$ & $5494(25.9)$ & $4766(22.5)$ \\
\hline Mean age, years (range) & $43.4(20-88)$ & $47.5(21-84)$ & $43.6(21-85)$ \\
\hline Mean BMI, $\mathrm{kg} / \mathrm{m}^{2}$ (SD) & $25.3(3.2)$ & $25.3(2.9)$ & $24.8(2.7)$ \\
\hline Mean PA score, index (SD) & NA & $1.77(0.22)$ & $2.36(0.21)$ \\
\hline Higher educationt, N (\%) & $645(8.6)$ & $762(13.9)$ & $821(17.2)$ \\
\hline Alcohol in past 2 weeks. N (\%) & $4489(60.1)$ & $3276(59.6)$ & 2895 (60.7) \\
\hline Current smoking, $\mathrm{N}(\%)$ & $3122(41.8)$ & 1695 (30.9) & 1023 (21.5) \\
\hline Use of BP medication, $\mathrm{N}(\%)$ & $445(6.0)$ & $453(8.3)$ & $245(5.1)$ \\
\hline Prevalent CVD, N (\%) & $192(2.6)$ & $286(5.2)$ & $153(3.2)$ \\
\hline \multicolumn{4}{|l|}{ Women } \\
\hline Number of persons, $\mathrm{N}(\%)$ & $7673(32.0)$ & $5060(21.1)$ & $7042(29.4)$ \\
\hline Mean age, years (range) & $44.4(13.9)$ & $47.6(14.8)$ & 44.5 (14.2) \\
\hline Mean BMI, $\mathrm{kg} / \mathrm{m}^{2}(\mathrm{SD})$ & $24.9(4.4)$ & $25.0(4.1)$ & $24.2(3.7)$ \\
\hline Mean PA score, index (SD) & NA & $1.57(0.15)$ & $2.07(0.20)$ \\
\hline Higher education†, N (\%) & $509(6.6)$ & $402(7.9)$ & $904(12.8)$ \\
\hline Alcohol in past 2 weeks. $\mathrm{N}(\%)$ & $2490(32.5)$ & $1623(32.1)$ & 2747 (39.0) \\
\hline Current smoking, N (\%) & $2962(38.6)$ & $1485(29.4)$ & $1907(27.1)$ \\
\hline Use of BP medication, $\mathrm{N}(\%)$ & $803(10.5)$ & $622(12.3)$ & $609(8.7)$ \\
\hline Prevalent CVD, N (\%) & $166(2.2)$ & $132(2.6)$ & $128(1.8)$ \\
\hline
\end{tabular}

\section{RESULTS}

After an average follow-up of 11 years, 1136 of 45186 individuals developed diabetes. In a subsample of 38413 individuals who had information on physical activity, 957 developed diabetes. Baseline characteristics of the participants who had valid information on the physical activity summary score are presented in table 1 .

\section{BMI, physical activity and diabetes risk}

Overall, there was a strong positive association $\left(\mathrm{P}_{\text {trend }}\right.$, $<0.001)$ between BMI and risk of developing diabetes in men and women (table 2). Compared to normal weight men, the risk of diabetes was threefold higher in men who were overweight (RR 3.21, 95\% CI 2.49 to 4.14) and ninefold among men who were obese (RR 8.93, 95\% CI 6.69 to 12). Women displayed an even stronger association, where those who were overweight and obese had RRs of 3.52 (95\% CI 2.63 to 4.73 ) and 9.97 (95\% CI 7.38 to 13), compared to normal weight women.

Men who were classified with a high physical activity summary score had a $35 \%$ lower risk of diabetes compared to men with a low score (RR $0.65,95 \%$ CI 0.51 to $0.84)$. The risk of diabetes was $24 \%$ lower among women with a high physical activity summary score compared to women with a low score (RR $0.76,95 \%$ CI 0.61 to 0.95 ).

Each measure of physical activity (frequency, duration, intensity, summary score) showed a gradual inverse association with diabetes risk in men and women (table 3). The inverse association was strongest with frequency in men $\left(\mathrm{P}_{\text {trend }}<0.001\right)$ and with frequency and duration in women $\left(\mathrm{P}_{\text {trend }}\right.$ 0.005). Men who reported being physically active four or more times a week had a $45 \%$ lower risk of developing diabetes (RR $0.55,95 \%$ CI 0.38 to 0.80 ) compared to men who reported no physical activity. The corresponding RR in women was 0.71 (95\% CI 0.52 to 0.96$)$. Women who reported physical activity with medium to high intensity had an RR of 0.62 (95\% CI 0.44 to 0.87 ).

We also assessed the potentially modifying effect of age on the association between physical activity and risk of diabetes, and found a $\mathrm{p}$ value for interaction of 0.92 among men and 0.03 among women. Stratified analyses among women showed that the association was largely confined to those aged $<60$ years (data not shown).

\section{Combined association of BMI and physical activity with diabetes risk}

Table 4 shows the combined association of BMI and physical activity with the risk of diabetes among men. Although there was no statistically significant interaction, obese men who reported no physical activity had 17 times higher risk of developing diabetes (RR 17, 95\% CI 9.52 to 30 ) than the reference group of normal weight men with a high physical activity summary score. On the other hand, obese men who were classified with a high physical activity summary score had an RR of 13 (95\% CI 6.92 to 26). The results showed a similar pattern in men who were overweight; if they reported no physical activity, they had an RR of 6.34 (95\% CI 3.65 to 11) compared to the reference group, whereas overweight men 
Table 2 RR of diabetes according to BMl and a physical activity summary score*, by gender

\begin{tabular}{|c|c|c|c|c|c|c|c|c|c|c|}
\hline & \multicolumn{5}{|l|}{ Men } & \multicolumn{5}{|c|}{ Women } \\
\hline & $\overline{\mathbf{C}}$ & Pers & $\mathbf{R R T}$ & RR‡ (95\% Cl) & p Value & $\overline{\mathbf{C}}$ & Pers. & $\mathbf{R R} \boldsymbol{R}$ & $\mathrm{RR} \ddagger(95 \% \mathrm{Cl})$ & p Value \\
\hline \multicolumn{11}{|l|}{$\mathrm{BMI}\left(\mathrm{kg} / \mathrm{m}^{2}\right)$} \\
\hline $14.5-24.9$ & 95 & 10988 & 1.00 & 1.00 (reference) & & 87 & 14629 & 1.00 & 1.00 (reference) & \\
\hline $25.0-29.9$ & 300 & 8838 & 3.10 & 3.21 (2.49 to 4.14$)$ & & 218 & 6821 & 3.50 & 3.52 (2.63 to 4.73$)$ & \\
\hline$\geq 30.0$ & 156 & 1384 & 9.01 & 8.93 (6.69 to 12$)$ & & 280 & 2525 & 9.91 & 9.97 (7.38 to 13$)$ & \\
\hline $\begin{array}{l}P_{\text {trend }} \\
\text { PA summan }\end{array}$ & y scor & & & & $<0.001$ & & & & & $<0.001$ \\
\hline Low & 235 & 7466 & 1.00 & 1.00 (reference) & & 226 & 7673 & 1.00 & 1.00 (reference) & \\
\hline Medium & 156 & 5494 & 0.72 & 0.80 (0.66 to 0.98$)$ & & 130 & 5060 & 0.73 & 0.81 (0.65 to 1.00$)$ & \\
\hline High & 86 & 4766 & 0.54 & 0.65 (0.51 to 0.84$)$ & & 124 & 7042 & 0.63 & 0.76 (0.61 to 0.95$)$ & \\
\hline$P_{\text {trend }}$ & & & & & $<0.001$ & & & & & 0.010 \\
\hline
\end{tabular}

*Summary score combining information on frequency, duration and intensity among those who were active $\geq 1$ a week (sex-specific medians defining medium and high activity). No activity and activity $<1$ a week were grouped as low activity.

†Adjusted for age (continuous); education ( $\leq 9$ years, $10-12$ years, $>12$ years; unknown); alcohol frequency in the past 2 weeks (no, $1-4, \geq 5$, abstainer; unknown); smoking (never, former, current, unknown); BP medication use (yes, no, unknown); prevalent CVD (yes, no, unknown). ¥Mutually adjusted for BMI and PA summary score in addition to covariates ${ }^{\dagger}$.

BMI, body mass index; BP, blood pressure; C, cases; CVD, cardiovascular disease; NA, not applicable; PA, physical activity; Pers., persons; $\mathrm{RR}$, risk ratio.

Table 3 RR of diabetes according to physical activity measures, by gender

\begin{tabular}{|c|c|c|c|c|c|c|c|c|}
\hline & \multicolumn{3}{|l|}{ Men } & \multirow[b]{2}{*}{ p Value } & \multicolumn{3}{|c|}{ Women } & \multirow[b]{2}{*}{ p Value } \\
\hline & $\overline{\mathbf{C}}$ & Pers. & $\mathrm{RR}^{*}(95 \% \mathrm{Cl})$ & & $\overline{\mathbf{C}}$ & Pers. & $\mathrm{RR}^{\star}(95 \% \mathrm{Cl})$ & \\
\hline \multicolumn{9}{|c|}{ Frequency per week } \\
\hline No activity & 73 & 1948 & 1.00 (reference) & & 97 & 1972 & 1.00 (reference) & \\
\hline$<1$ per week & 162 & 5518 & $0.96(0.73$ to 1.25$)$ & & 129 & 5701 & $0.83(0.64$ to 1.07$)$ & \\
\hline 1 per week & 110 & 4610 & 0.77 (0.57 to 1.03$)$ & & 124 & 5778 & 0.70 (0.53 to 0.92$)$ & \\
\hline 2-3 per week & 98 & 4062 & 0.84 (0.62 to 1.13$)$ & & 94 & 4663 & 0.67 (0.50 to 0.88$)$ & \\
\hline$\geq 4$ per week & 45 & 1881 & 0.55 (0.38 to 0.80$)$ & & 63 & 2280 & 0.71 (0.52 to 0.96$)$ & \\
\hline \multicolumn{9}{|c|}{ Minutes per session } \\
\hline No activity & 73 & 1948 & 1.00 (reference) & & 97 & 1972 & 1.00 (reference) & \\
\hline $1-15$ & 37 & 934 & 0.89 (0.61 to 1.29$)$ & & 46 & 1384 & 0.73 (0.52 to 1.02$)$ & \\
\hline $16-30$ & 119 & 3509 & 0.94 (0.71 to 1.25$)$ & & 121 & 5350 & 0.65 (0.50 to 0.86$)$ & \\
\hline $31-60$ & 120 & 5208 & 0.75 (0.56 to 1.00$)$ & & 128 & 6349 & 0.75 (0.57 to 0.97$)$ & \\
\hline$\geq 60$ & 66 & 3081 & 0.70 (0.51 to 0.98$)$ & & 28 & 1396 & 0.74 (0.49 to 1.12$)$ & \\
\hline $\begin{array}{l}P_{\text {trend }} \\
\text { Intensity }\end{array}$ & & & 0.011 & & & & 0.056 & \\
\hline No activity & 73 & 1948 & 1.00 (reference) & & 97 & 1972 & 1.00 (reference) & \\
\hline Low & 206 & 5149 & $0.91(0.70$ to 1.18$)$ & & 279 & 9061 & $0.83(0.66$ to 1.04$)$ & \\
\hline Medium/high & 133 & 7272 & $0.71(0.53$ to 0.95$)$ & & 57 & 5285 & $0.62(0.44$ to 0.87$)$ & \\
\hline $\mathrm{P}_{\text {trend }}$ & & & & 0.011 & & & & 0.005 \\
\hline
\end{tabular}

with a high physical activity summary score had an RR of 4.31 (95\% CI 2.39 to 7.78$)$.

Obese, inactive women had an RR of 15 (95\% CI 9.18 to 25) of developing diabetes compared to normal weight women classified with a high summary score. Obese women with a high physical activity summary score had an RR of 13 (95\% CI 7.42 to 21). For overweight women, the risk was highest among the inactive (RR 5.54, 95\% CI 3.36 to 9.13) and lowest among the most physically active (RR 4.32, 95\% CI 2.55 to 7.32) (table 5). No statistically significant interaction was found.

\section{DISCUSSION}

In this prospective longitudinal study, we found that both BMI and physical activity were independently associated with risk of diabetes. Obese individuals had a 
Table 4 RR of diabetes according to joint categories of BMI and physical activity among men

\begin{tabular}{|c|c|c|c|c|}
\hline \multirow[b]{2}{*}{ Physical activity summary score ${ }^{*}$} & \multicolumn{3}{|c|}{ Body mass index $\left(\mathrm{kg} / \mathrm{m}^{2}\right)$} & \multirow[b]{2}{*}{ p Value } \\
\hline & $14.5-24.9$ & 25.0-29.9 & $\geq 30.0$ & \\
\hline \multicolumn{5}{|l|}{ High } \\
\hline Number of cases & 14 & 50 & 22 & \\
\hline Number of persons & 2777 & 1785 & 204 & \\
\hline $\mathrm{RR} \dagger(95 \% \mathrm{Cl})$ & 1.00 (reference) & 4.31 (2.39 to 7.78$)$ & $13(6.92$ to 26$)$ & \\
\hline \multicolumn{5}{|l|}{ Medium } \\
\hline Number of cases & 25 & 90 & 41 & \\
\hline Number of persons & 2749 & 2397 & 348 & \\
\hline $\mathrm{RR} \dagger(95 \% \mathrm{Cl})$ & $1.55(0.80$ to 2.97$)$ & 5.12 (2.92 to 9.00$)$ & 15 (8.03 to 27$)$ & \\
\hline \multicolumn{5}{|l|}{ Low } \\
\hline Number of cases & 40 & 124 & 71 & \\
\hline Number of persons & 3662 & 3209 & 595 & \\
\hline $\mathrm{RR} \dagger(95 \% \mathrm{Cl})$ & $2.29(1.24$ to 4.21$)$ & $6.34(3.65$ to 11$)$ & 17 (9.52 to 30$)$ & \\
\hline$P_{\text {trend }}$ & & & & $<0.001$ \\
\hline$P_{\text {interaction }}$ & & & & 0.108 \\
\hline \multicolumn{5}{|c|}{$\begin{array}{l}\text { *Summary score combining information on frequency, duration and intensity among those who were active } \geq 1 \text { a week (sex-specific medians } \\
\text { defining medium and high activity). No activity and activity }<1 \text { a week were grouped as low activity. } \\
\text { †Adjusted for age (continuous); education ( } \leq 9 \text { years, } 10-12 \text { years, }>12 \text { years; unknown); alcohol frequency in the past } 2 \text { weeks (no, } 1-4, \geq 5 \text {, } \\
\text { abstainer; unknown); smoking (never, former, current, unknown); BP medication use (yes, no, unknown); prevalent CVD (yes, no, unknown). } \\
\text { BMI, body mass index; BP, blood pressure; C, cases; CVD, cardiovascular disease; NA, not applicable; PA, physical activity; Pers., persons; } \\
\text { RR, risk ratio. }\end{array}$} \\
\hline
\end{tabular}

Table 5 RR of diabetes according to joint categories of BMI and physical activity among women

\begin{tabular}{|c|c|c|c|c|}
\hline \multirow[b]{2}{*}{ Physical activity summary score* } & \multicolumn{3}{|c|}{ Body mass index $\left(\mathrm{kg} / \mathrm{m}^{2}\right)$} & \multirow[b]{2}{*}{ p Value } \\
\hline & $14.5-24.9$ & $25.0-29.9$ & $\geq \mathbf{3 0 . 0}$ & \\
\hline \multicolumn{5}{|l|}{ High } \\
\hline Number of cases & 20 & 51 & 53 & \\
\hline Number of persons & 4649 & 1853 & 540 & \\
\hline $\mathrm{RR} \dagger(95 \% \mathrm{Cl})$ & 1.00 (reference) & 4.32 (2.55 to 7.32$)$ & $13(7.42$ to 21$)$ & \\
\hline \multicolumn{5}{|l|}{ Medium } \\
\hline Number of cases & 17 & 49 & 64 & \\
\hline Number of persons & 2932 & 1555 & 573 & \\
\hline $\mathrm{RR} \dagger(95 \% \mathrm{Cl})$ & 1.25 (0.65 to 2.37$)$ & 4.32 (2.53 to 7.37$)$ & 13 (7.71 to 22$)$ & \\
\hline \multicolumn{5}{|l|}{ Low } \\
\hline Number of cases & 33 & 79 & 114 & \\
\hline Number of persons & 4489 & 2231 & 953 & \\
\hline $\mathrm{RR}+(95 \% \mathrm{Cl})$ & 1.80 (1.03 to 3.14$)$ & 5.54 (3.36 to 9.13$)$ & 15 (9.18 to 25$)$ & \\
\hline$P_{\text {trend }}$ & & & & $<0.001$ \\
\hline$P_{\text {interaction }}$ & & & & 0.223 \\
\hline \multicolumn{5}{|c|}{$\begin{array}{l}\text { *Summary score combining information on frequency, duration and intensity among those who were active } \geq 1 \text { a week (sex-specific medians } \\
\text { defining medium and high activity). No activity and activity }<1 \text { a week were grouped as low activity. } \\
\text { †Adjusted for age (continuous); education ( } \leq 9 \text { years, } 10-12 \text { years, }>12 \text { years; unknown); alcohol frequency in the past } 2 \text { weeks (no, } 1-4, \geq 5 \text {, } \\
\text { abstainer; unknown); smoking (never, former, current, unknown); BP medication use (yes, no, unknown); prevalent CVD (yes, no, unknown). } \\
\text { BMl, body mass index; BP, blood pressure; C, cases; CVD, cardiovascular disease; NA, not applicable; PA, physical activity; Pers., persons; } \\
\text { RR, risk ratio. }\end{array}$} \\
\hline
\end{tabular}

ninefold higher risk than normal weight individuals. The most physically active individuals had approximately two-thirds the risk of diabetes of those who were inactive. The combined effect of BMI and physical activity yielded a 17-fold higher risk of diabetes among obese and inactive men. Obese men who reported high activity had an 13 times higher risk than their normal weight and highly active peers. For women the associations were similar, although differences in RRs were somewhat smaller. Women who were obese and inactive had a 15-fold higher risk than normal weight and highly active women, whereas the risk was 13 times higher among highly active, obese women.

The results from this study are generally in line with several previous studies where adiposity is shown to be an important predictor for developing diabetes, especially in women, ${ }^{1-5}$ Results from the Nurses' Health Study in the USA showed that $91 \%$ of the cases of diabetes could be attributed to a high-risk lifestyle, with a BMI above $25 \mathrm{~kg} / \mathrm{m}^{2}$ and low physical activity levels, and 
that the strength of the association with BMI was stronger than that with physical activity. ${ }^{1}$

Studies have also shown that physical activity decreases the risk of diabetes, even after controlling for BMI. ${ }^{20-22}$ This has been supported by randomised trials where lifestyle interventions including increased physical activity have shown beneficial effects on diabetes. The Finnish Diabetes Prevention Study ${ }^{23}$ found that the intervention group had less than half the risk of diabetes compared to the control group, and the Diabetes Prevention Program $^{24}$ in the USA found that lifestyle modification was more beneficial than metformin therapy in reducing the risk of diabetes. Moreover, the Da Quing diabetes study $^{25}$ in China divided participants with impaired glucose tolerance into one control group and three intervention groups, diet, exercise and diet plus exercise, and found $31 \%, 46 \%$ and $42 \%$ reduction in the development of diabetes compared to controls, respectively.

The hypothesis that overweight or obese individuals who engage in regular physical activity may cancel out the adverse effects of adiposity has been tested for cardiovascular disease with inconclusive results. ${ }^{26-28}$ Tuomilehto $e t a l^{8}$ applied this hypothesis on the risk of diabetes type 2 and found that physical activity reduced the risk of developing diabetes even if the individuals did not achieve the targeted weight loss, which is consistent with our results. Later, Rana et a $\bar{l}$ and Weinstein et $a l^{15}$ have examined the combined effect of physical activity and BMI on the risk of developing diabetes in women, and found that physical activity only modestly affected the influence of BMI on diabetes risk. The results from this study are consistent with those in these two studies.

\section{Biological plausibility of the observed results}

Physical activity may reduce adiposity-induced diabetes by changing body composition, such as the reduction of total and abdominal fat, and by improving insulin resistance, cholesterol levels, blood pressure and glucose tolerance. The somewhat mitigating effect from physical activity on diabetes among obese individuals, observed in our data, is supported by the plausibility that individuals with high fitness levels have a metabolic profile related to better weight control and to protection against insulin resistance. ${ }^{24} 25$ Physical activity seems to improve insulin sensitivity particularly well when insulin sensitivity is impaired, such as in individuals with obesity. ${ }^{26}$ Underlying mechanisms of the benefits of physical activity in relation to diabetes also include improvements in endothelial vasodilator function and left ventricular diastolic function. ${ }^{27}$ Further, acute effects from physical exercise, such as increased flow of substrates and secretion of hormones and increased glucose uptake by muscle contractions, ${ }^{29}$ may play important mechanistic roles in the reduction of diabetes risk. Also, postexercise induced hypotension, which in turn facilitates sustained vasodilation, has been suggested as an important mechanism that improves glucose uptake by skeletal muscles in patients with diabetes. ${ }^{28}$

\section{Strengths and limitations}

The main strengths of this study include the prospective longitudinal design and the large sample size with a high number of diabetes cases, providing sufficient statistical power to conduct analyses of the combined effect of BMI and physical activity. Moreover, the HUNT study covers a total adult population within a geographical area and has a high participation rate. The population is stable and homogeneous, with low in-migration and out-migration. ${ }^{30}$

The precision of the estimated associations and the consistent gradual reduction in diabetes risk with increasing level of physical activity suggest that the results are not explained by chance. However, biased estimates due to confounding by unmeasured and unknown factors cannot be excluded in this type of study. Other studies have found dietary factors ${ }^{1}{ }^{731}$ and family history of diabetes ${ }^{32}$ to be important risk factors; however we did not have sufficient information to adjust for these potential confounders.

Misclassification of the diabetes outcome could be present since outcome was defined as a positive response to the question "Do you have or have you had diabetes". However, this question has been validated by comparing the questionnaire answers to medical records, and the diagnosis was verified in $96 \%$ of the cases. ${ }^{16}$ Another source of bias could be that we were unable to differentiate between diabetes type 1 and diabetes type 2. Still, the large majority $(\sim 85 \%)$ of cases are most likely to be diabetes type 2 , and the ratio of type 2 vs type 1 should not be differential between the exposure groups. Also, diabetes type 1 is a polygenic disorder not driven by inactivity and adiposity to the same extent as diabetes type $2 .^{33}$

Although questionnaire-based information on physical activity is relatively easily accessible and inexpensive, it allows for subjective interpretation of the questions and individual perception of the activity, and misclassification could be influenced by factors such as age, social context and seasonal variation. However, validation studies have shown that questionnaires may be useful in classifying people into broad categories of physical activity. ${ }^{34}$ Reliability and validity of self-reported physical activity as reported in HUNT1 have been assessed, and found to provide a useful measure of leisure time physical activity. ${ }^{35}$ Still, the exact amount of physical activity that is needed to have a substantial impact on insulin and blood glucose levels goes beyond the limitations of a subjective activity questionnaire and should be answered by more objective measures. Questionnaire-based information has also been known to overestimate the general physical activity level, and this could result in an underestimation of the effect of physical activity on diabetes risk. ${ }^{36}$ The equation for the physical activity summary score was developed on the 
basis that frequency, duration and intensity should be equally weighted, and changing the weighting for the various components could possibly alter the findings. We based the equation on results from table 3, indicating that associations between the different physical activity components and diabetes risk were of largely similar magnitude. The summary score has been used in previous studies. ${ }^{1718}$

BMI may overestimate adiposity in short and heavy individuals with lean body mass, and could underestimate adiposity in those with less lean body mass. ${ }^{37}$ Another problem is that the correspondence between BMI and adiposity differs between men and women with similar BMI, where women would most likely have a greater percentage of adiposity. ${ }^{38}$ Hence, misclassification of BMI could have influenced the results since athletic people with high muscle mass, misclassified as being overweight or obese, would probably report having high levels of physical activity. Therefore, the protective effect of physical activity in the combined analyses could be overestimated, especially in men.

We are aware that some people were lost to follow-up between HUNT1 and HUNT2 due to death, migration or non-participation. However, for this to bias our estimates, loss to follow-up must have been differential between exposure categories. It is conceivable that lean and physically active people to a greater extent were alive and willing to participate in HUNT2 than those who were obese and inactive. This could have underestimated the effects of adiposity and physical activity in our data. Comparisons between participants and nonparticipants in the HUNT studies have shown that nonparticipants had higher prevalence of cardiovascular disease, diabetes and psychiatric disorders, lower socioeconomic status and higher mortality than participants. ${ }^{30}$

\section{CONCLUSION}

This study shows that overweight and obesity are associated with a substantially increased risk of diabetes in men and women, particularly among those who also reported being physically inactive. Although there was little evidence for a compensatory effect of physical activity on overweight and obese individuals, high levels of physical activity were associated with a lower risk of diabetes within all categories of BMI.

Acknowledgements Nord-Trøndelag Health Study (The HUNT Study) is a collaboration between HUNT Research Centre (Faculty of Medicine, Norwegian University of Science and Technology NTNU), Nord-Trøndelag County Council and The Norwegian Institute of Public Health.

Contributors KVH and TILN contributed to the conception and design of the work. TILN contributed to the collection and assembly of data. All authors (KVH, JSS and TILN) contributed to the data analyses and interpretation of data, in drafting and revising it critically for important intellectual content, and in giving final approval of the version to be published. All authors agreed to be accountable for all aspects of the work in ensuring that questions relating to the accuracy and integrity of any part of the work are appropriately investigated and resolved.
Funding This research received no specific grant from any funding agency in the public, commercial, or not-for-profit sectors.

Competing interests None declared.

Ethics approval Norwegian Regional Committee for Ethics in Medical Research.

Provenance and peer review Not commissioned; externally peer reviewed.

Data sharing statement No additional data are available.

Open Access This is an Open Access article distributed in accordance with the Creative Commons Attribution Non Commercial (CC BY-NC 4.0) license, which permits others to distribute, remix, adapt, build upon this work noncommercially, and license their derivative works on different terms, provided the original work is properly cited and the use is non-commercial. See: http:// creativecommons.org/licenses/by-nc/4.0/

\section{REFERENCES}

1. Hu FB, Manson JE, Stampfer MJ, et al. Diet, lifestyle, and the risk of type 2 diabetes mellitus in women. N Engl J Med 2001;345:790-7.

2. Hu FB, Willett WC, Li T, et al. Adiposity as compared with physical activity in predicting mortality among women. $N$ Engl $J$ Med 2004;351:2694-703.

3. Chan JM, Rimm EB, Colditz GA, et al. Obesity, fat distribution, and weight gain as risk factors for clinical diabetes in men. Diabetes Care 1994;17:961-9.

4. Colditz GA, Willett WC, Rotnitzky A, et al. Weight gain as a risk factor for clinical diabetes mellitus in women. Ann Intern Med 1995;122:481-6.

5. Rana JS, Li TY, Manson JE, et al. Adiposity compared with physical inactivity and risk of type 2 diabetes in women. Diabetes Care 2007;30:53-8.

6. Eriksson KF, Lindgarde F. Prevention of type 2 (non-insulin-dependent) diabetes mellitus by diet and physical exercise. The 6-year Malmo feasibility study. Diabetologia 1991;34:891-8.

7. Pan XR, Li GW, Hu YH, et al. Effects of diet and exercise in preventing NIDDM in people with impaired glucose tolerance. The Da Qing IGT and Diabetes Study. Diabetes Care 1997;20:537-44.

8. Tuomilehto J, Lindström J, Eriksson JG, et al. Prevention of type 2 diabetes mellitus by changes in lifestyle among subjects with impaired glucose tolerance. N Engl J Med 2001;344:1343-50.

9. Knowler WC, Barrett-Connor E, Fowler SE, et al. Reduction in the incidence of type 2 diabetes with lifestyle intervention or metformin N Engl J Med 2002;346:393-403.

10. LaMonte MJ, Blair SN, Church TS. Physical activity and diabetes prevention. J Appl Physiol 2005;99:1205-13.

11. O'Gorman DJ, Karlsson HK, McQuaid S, et al. Exercise training increases insulin-stimulated glucose disposal and GLUT4 (SLC2A4) protein content in patients with type 2 diabetes. Diabetologia 2006;49:2983-92.

12. Boulé NG, Haddad E, Kenny GP, et al. Effects of exercise on glycemic control and body mass in type 2 diabetes mellitus: a meta-analysis of controlled clinical trials. JAMA 2001;286:1218-27.

13. Tjønna AE, Lund Nilsen TI, Slørdahl SA, et al. The association of metabolic clustering and physical activity with cardiovascular mortality: the HUNT study in Norway. J Epidemiol Community Health 2010;64:690-5.

14. Vatten LJ, Nilsen TI, Romundstad PR, et al. Adiposity and physical activity as predictors of cardiovascular mortality. Eur J Cardiovasc Prev Rehabil 2006;13:909-15.

15. Weinstein AR, Sesso HD, Lee Intramuscular, et al. Relationship of physical activity vs body mass index with type 2 diabetes in women. JAMA 2004;292:1188-94.

16. Midthjell $\mathrm{K}$, Holmen J, Bjorndal $\mathrm{A}$, et al. Is questionnaire information valid in the study of a chronic disease such as diabetes? The Nord-Trondelag diabetes study. J Epidemiol Community Health 1992;46:537-42.

17. Nilsen TI, Romundstad PR, Vatten LJ. Recreational physical activity and risk of prostate cancer: a prospective population-based study in Norway (the HUNT study). Int J Cancer 2006;119:2943-7.

18. Augestad LB, Schei B, Forsmo S, et al. Healthy postmenopausal women-physical activity and forearm bone mineral density: the Nord-Trondelag health survey. J Women Aging 2006;18:21-40

19. Zou G. A modified poisson regression approach to prospective studies with binary data. Am J Epidemiol 2004;159:702-6. 
20. Helmrich SP, Ragland DR, Leung RW, et al. Physical activity and reduced occurrence of non-insulin-dependent diabetes mellitus. N Engl J Med 1991;325:147-52.

21. Manson JE, Rimm EB, Stampfer MJ, et al. Physical activity and incidence of non-insulin-dependent diabetes mellitus in women. Lancet 1991;338:774-8.

22. Ivy JL. Role of exercise training in the prevention and treatment of insulin resistance and non-insulin-dependent diabetes mellitus. Sports Med 1997;24:321-36.

23. Lee CD, Blair SN, Jackson AS. Cardiorespiratory fitness, body composition, and all-cause and cardiovascular disease mortality in men. Am J Clin Nutr 1999;69:373-80.

24. Fogelholm M. Physical activity, fitness and fatness: relations to mortality, morbidity and disease risk factors. A systematic review. Obes Rev 2010;11:202-21.

25. Hamer M, Stamatakis E. Physical activity and risk of cardiovascular disease events: inflammatory and metabolic mechanisms. Med Sci Sports Exerc 2009:41:1206-11.

26. Gill JM, Al-Mamari A, Ferrell WR, et al. Effects of prior moderate exercise on postprandial metabolism and vascular function in lean and centrally obese men. J Am Coll Cardiol 2004;44:2375-82.

27. Stewart KJ. Exercise training and the cardiovascular consequences of type 2 diabetes and hypertension: plausible mechanisms for improving cardiovascular health. JAMA 2002;288:1622-31.

28. Halliwill JR, Buck TM, Lacewell AN, et al. Postexercise hypotension and sustained postexercise vasodilatation: what happens after we exercise? Exp Physiol 2013;98:7-18.
29. Rankinen $\mathrm{T}$, Bouchard C. Invited commentary: physical activity, mortality, and genetics. Am J Epidemiol 2007;166:260-2.

30. Krokstad S, Langhammer A, Hveem K, et al. Cohort profile: the HUNT study, Norway. Int J Epidemiol 2013;42:968-77.

31. Salmerón J, Manson JE, Stampfer MJ, et al. Dietary fiber, glycemic load, and risk of non-insulin-dependent diabetes mellitus in women. JAMA 1997;277:472-7.

32. Thomas F, Balkau B, Vauzelle-Kervroedan F, et al. Maternal effect and familial aggregation in NIDDM. The CODIAB study. CODIAB-INSERM-ZENECA study group. Diabetes 1994;43:63-7.

33. Atkinson MA, Eisenbarth GS, Michels AW. Type 1 diabetes. Lancet 2014;383:69-82.

34. Shephard RJ. Limits to the measurement of habitual physical activity by questionnaires. Br J Sports Med 2003;37:197-206; discussion 206.

35. Kurtze N, Rangul V, Hustvedt BE, et al. Reliability and validity of self-reported physical activity in the Nord-Trondelag Health study: HUNT 1. Scand J Public Health 2008;36:52-61.

36. Rzewnicki R, Vanden Auweele Y, De Bourdeaudhuij I. Addressing overreporting on the International Physical Activity Questionnaire (IPAQ) telephone survey with a population sample. Public Health Nutr 2003;6:299-305.

37. Romero-Corral A, Somers VK, Sierra-Johnson J, et al. Accuracy of body mass index in diagnosing obesity in the adult general population. Int J Obes (Lond) 2008;32:959-66.

38. Rothman KJ. BMI-related errors in the measurement of obesity. Int J Obes (Lond) 2008;32(Suppl 3):S56-9. 